\title{
Petite bibliographie sur l'analyse du discours philosophique
}

Frédéric Cossutta et Dominique Maingueneau

\section{(2) OpenEdition \\ 1 Journals}

Édition électronique

URL : http://journals.openedition.org/aad/3205

DOI : 10.4000/aad.3205

ISSN : 1565-8961

Éditeur

Université de Tel-Aviv

Référence électronique

Frédéric Cossutta et Dominique Maingueneau, «Petite bibliographie sur l'analyse du discours

philosophique », Argumentation et Analyse du Discours [En ligne], 22 | 2019, mis en ligne le 15 avril 2019 consulté le 23 septembre 2019. URL : http://journals.openedition.org/aad/3205 ; DOI : 10.4000/aad. 3205

Ce document a été généré automatiquement le 23 septembre 2019.

\section{c) (†) $९$}

Argumentation \& analyse du discours est mis à disposition selon les termes de la licence Creative Commons Attribution - Pas d'Utilisation Commerciale - Pas de Modification 4.0 International. 


\title{
Petite bibliographie sur l'analyse du discours philosophique
}

\author{
Frédéric Cossutta et Dominique Maingueneau
}

Cette courte bibliographie - où ne figurent pas les articles du présent numéro d' Argumentation et Analyse du Discours - ne peut prétendre recenser tous les travaux qui ont pris pour objet, partiellement ou totalement, la philosophie envisagée dans sa dimension linguistique, textuelle ou discursive. Elle met l'accent sur ceux qui relèvent d'une perspective d'analyse du discours, ou en sont proches. Nous sommes bien conscients que le fait de ranger telle ou telle publication dans telle ou telle rubrique comporte une bonne part d'arbitraire et que ces rubriques elles-mêmes sont discutables. En outre, beaucoup de textes auraient pu être placés dans plusieurs rubriques, et celles-ci auraient pu être subdivisées plus avant pour tenir compte de la complexité des problématiques. Nous espérons néanmoins qu'en dépit de ses évidentes imperfections elle sera utile. Nous avons pris le parti de ranger dans chaque rubrique les publications en suivant l'ordre chronologique; il nous a semblé que cela donnait une vision plus réaliste de l'évolution des problématiques.

\section{L'analyse du discours philosophique}

\subsection{Aspects généraux}

Cossutta, Frédéric. 1989. Éléments pour la lecture des textes philosophiques (Paris : Bordas)

Schneider, Ulrich Johannes. 1990. Die Vergangenheit des Geistes. Eine Archäologie der Philosophiegeschichte (Frankfurt a. M. : Suhrkamp)

Cossutta, Frédéric. 1994. "Catégories discursives et analyse du discours philosophique ", Moirand, Sophie, Magid Ali Bouacha, Jean-Claude Beacco \& André Collinot (éds.). Parcours linguistiques de discours spécialisés (Berne : Peter Lang), 349-360

Cossutta, Frédéric (éd.). 1995. « L'analyse du discours philosophique », Langages 119

Cossutta, Frédéric. 1995. « Présentation », Langages 119, 5-11 

12-39 discursive ", Langages 119, 40-62 der Philosophiegeschichte im 20. Jahrhundert », Klaus-Dieter Eichler \& Volker Caysa (eds). Philosophiegeschichte und Hermeneutik (Leipzig: Leipziger Universitätsverlag), 46-69

9 Cossutta, Frédéric. 1998. «L'analyse du discours philosophique », Mattéi, Jean-François (éd.). Encyclopédie philosophique universelle, t. IV, Le discours philosophique (Paris : PUF), 1792-1810

10 Maingueneau, Dominique. 1998. "Textes philosophique, énonciation et institution », Mattéi, Jean-François (éd.). Encyclopédie philosophique Universelle, t. IV, Le Discours philosophique (Paris : PUF), 1810-1820

11 Rastier, François. 2001. "L'Être naquit dans le langage ", Methodos [En ligne] URL : http://journals.openedition.org/methodos/206 ; DOI : 10.4000/methodos.206

12 Cossutta, Frédéric. 2004. «Catégories descriptives, catégories interprétatives pour le texte philosophique ", Ali-Bouacha, Magid, Jean-Michel Adam \& Jean-Blaise Grize (éds). Texte et discours: catégories pour l'analyse (Dijon: Éditions Universitaires de Dijon), 190-213

13 Temmar, Malika. 2007. "Analyse du discours et philosophie: croisements réciproques ", Bonnafous, Simone \& Malika Temmar (éds). Analyse du discours et sciences humaines et sociales (Paris : Ophrys)

14 Vallespir, Mathilde, Lia Kurts-Woeste \& Marie-Albane Watine (éds). 2012. La Violence du logos : entre sciences du texte, philosophie et littérature (Paris : Garnier)

15 Maingueneau, Dominique. 2015. La philosophie comme institution discursive (Limoges: Lambert-Lucas)

16 Vallespir, Mathilde. 2015. «L'ombre portée de la philosophie sur l'analyse du discours : paratopie et négativité ", Angermüller, Johannes \& Gilles Philippe (éds). Analyse du discours et dispositifs d'énonciation. Autour des travaux de Dominique Maingueneau (Limoges : Lambert-Lucas), 71-78

17 Bordron, Jean-François. 2016. Le discours spéculatif. Approche sémiotique (Limoge: Lambert-Lucas)

18 Cossutta, Frédéric. 2017. "Des idéalités mathématiques aux idéalités philosophiques », Association des Amis de Dominique et Jean-Toussaint Desanti [En ligne: http:// desanti.huma-num.fr/associationdesanti/node/73]

\subsection{La philosophie comme discours constituant}

Maingueneau, Dominique \& Frédéric Cossutta. 1995. "L'Analyse des discours constituants », Langages 117, 112-125 Maingueneau, Dominique. 1999. "Analyzing self-constituting discourses ", Discourse studies 1 (2), 175-199 
Cossutta, Frédéric. 2003. "Discours littéraire, discours philosophique : deux formes d'auto-constitution ", Amossy, Ruth \& Dominique Maingueneau (éds). L'analyse du discours dans les études littéraires (Toulouse : Presses Universitaires du Mirail), 417-428 Cossutta, Frédéric, 2005. «Discours philosophique, discours littéraire : le même et l'autre », Rue Descartes 50, « L'écriture des philosophes », 6-20

Maingueneau, Dominique. 2007. "Clarté du texte, discours constituants et cadre herméneutique », Denis, Delphine (éd.), L'obscurité. Langage et herméneutique sous l'Ancien régime (Louvain : Academia Bruylant), 13-23

Cossutta, Frédéric. 2015. "Les discours constituants vingt ans après ", Angermüller, Johannes \& Gilles Philippe (éds). Analyse du discours et dispositifs d'énonciation. Autour des travaux de Dominique Maingueneau (Limoges : Lambert-Lucas), 61-70

Maingueneau, Dominique. 2017. «Discours philosophique et discours religieux: la question du Thesaurus ", Agular, José, Lucile Cadet, Catherine Muller \& Véronique Rivière (éds). L'enseignant et le chercheur au cœur des discours et des actions. Mélanges offerts à Francine Cicurel (Paris : Riveneuve Éditions), 95-108

26 Maingueneau, Dominique. 2017. "Pseudonymie et discours constituants », Martens, David (éd.). La pseudonymie dans la littérature française. De François Rabelais à Eric Chevillard, (Rennes : P. U. de Rennes), 311-322

\title{
2. La philosophie dans sa dimension biographique et sociale
}

\subsection{Relations entre vie et œuvre}

\author{
« Biographies ». 1990. Les cahiers philosophiques 19
}

«Vies philosophiques ». 1999. Critique 627-628

Clément, Bruno. 2005. "Derrida: la Vie et l'đEuvre» (en anglais). Substance 106, « Jacques Derrida 1930-2004, à Counter-Obituary », 35-37

Clément, Bruno. 2008. "L'autobiographie de l'autre », Les transformateurs Lyotard (Paris : Sens et Tonka), 63-78

31 Clément, Bruno. 2010. "D’une autobiographie sans sujet - Levinas lecteur de Leiris ", Cohen-Levinas, Danielle (éd.). Le Souci de l'art chez Emmanuel Levinas (Paris : Éditions Manucius), 139-150

2 Clément, Bruno \& Christian Trottmann (éds). 2010. Vie philosophique et Vies de philosophes, (Paris : Sens \& Tonka)

33 Clément, Bruno. 2010. «Kant est-il mort comme il a vécu? A-t-il vécu comme il a écrit?", Clément, Bruno \& Christian Trottmann (éds.). Vie philosophique et Vies de philosophes (Paris : Sens \& Tonka)

4 Clément, Bruno. 2012. "Ce que la méthode doit à la vie», Cossutta, Delormas \& Maingueneau (éds.), 53-70

5 Cossutta, Frédéric, Pascale Delormas \& Dominique Maingueneau (éds). 2012. La Vie à l'œuvre, le biographique dans le discours philosophique (Limoges : Lambert-Lucas)

Bordron, Jean-François. 2012. "Maine de Biran et le diagramme de la subjectivité ", Cossutta, Delormas, Maingueneau (éds.), 87-106 
Cossutta, Frédéric. 2012. «Le statut du biographique dans le discours philosophique », Cossutta, Delormas \& Maingueneau (éds.), 121-152.

Delormas, Pascale. 2012. "Les Confessions disent-elles quelque chose de Rousseau?", Cossutta, Delormas \& Maingueneau (éds), 71-86

Giolito, Christophe. 2012. "Le discours d'Auguste Comte sur Clotilde de Vaux", Cossutta, Delormas \& Maingueneau (éds), 107-121

Maingueneau, Dominique. 2012. «La biographie des philosophes dans une perspective d'analyse du discours », Cossutta, Delormas \& Maingueneau (éds), 21-36

Ribard, Dinah. 2012. "Vies de philosophes et situation de la philosophie à l'époque moderne », Cossutta, Delormas \& Maingueneau (éds), 37-52

\subsection{Approches sociologiques et socio-discursives}

Collins, Randall. 1998. The Sociology of Philosophies. A Global Theory of Intellectual Change (Cambridge, Massachusetts, and London : The Belknap Press of Harvard U. P.)

3 Faye, Jean-Pierre. 1998. « Texte et idéologie », Encyclopédie philosophique universelle T. IV, ch. $\mathrm{n}^{\circ} 114,1946-1965$

4 Angermüller, Johannes. 2003. «Discours et champs intellectuels : l'antagonisme entre 'humanistes' et 'prophètes' et le discours des sciences humaines dans les années 60 et 70 », Amossy, Ruth, Dominique Maingueneau (éds). L'analyse du discours dans les études littéraires (Toulouse : P. U. du Mirail), 83-92

Dosse, François. 2003. La marche des idées. Histoire des intellectuels-Histoire intellectuelle (Paris : La Découverte)

Pinto, Louis. 2007. La vocation et le métier de philosophe. Pour une sociologie de la philosophie dans la France contemporaine (Paris : Seuil)

Delormas, Pascale. 2010. «République des lettres et positionnement paratopique de Jean-Jacques Rousseau », Moreira de Mello, Maria Celina \& Pedro Paulo Garcia Ferreira Catharina (éds.), Cenas da Literatura Moderna (Rio de Janeiro : 7 Letras), 19-41

Fabiani, Jean-Louis. 2010. Qu'est-ce qu'un philosophe français? La vie sociale des concepts (1880-1980) (Paris : Editions de l'EHESS)

Angermuller, Johannes. 2013. Le champ de la théorie. Essor et déclin du structuralisme en France (Paris : Hermann)

50 Pinto, Louis. 2014. Sociologie et philosophie : libres échanges. Bourdieu, Durkheim, Foucault, Sartre... (Paris : Ithaque)

1 Bianco, Giuseppe. 2015. Après Bergson : portrait de groupe avec philosophe (Paris : PUF)

Delormas, Pascale. 2017. «De la plume à l'herbe. Les promenades de Rousseau, métaphores d'un positionnement discursif », M. Merino García, M. Ledesma Pedraz, E. Medina Arjona, M. Olivares Palacios, Ma J. Sueza Espejo, Ma L. Torre Montes (éds.). L'appréciation langagière de la nature : le naturel, le texte et l'artifice (Jaen : Université de Jaen), 99-104 


\section{La textualité philosophique}

\subsection{La philosophie et la langue}

53 Le discours philosophique, troisième partie : Les formes de la philosophie, Mattéi, JeanFrançois (éd.). Encyclopédie philosophique universelle, T. IV (Paris, PUF, 1998) : Deuxième partie, « La Diffusion de la philosophie », Section 1 : Les chemins de la traduction, chap. $\mathrm{n}^{\circ} 54$ à 68, 976-1200

54 (Coll.) « Ce que les philosophes disent de leur langue ». 1999. Rue Descartes 26

55 Maurel, Jean. 2001. "L'idiome du philosophe », Cahiers philosophiques 89, "Styles et genres en philosophie », 9-22

56 Loraux, Patrice, François Noudelman, Antonia Soulez \& Frédéric Cossutta. 2005. «Le langage des philosophes » (transcription d'un entretien sur France Culture), Rue Descartes 50, 119-126

57 Maingueneau, Dominique. 2005. «Code langagier et scène d'énonciation philosophique », Rue Descartes 50, 21-33

Clément, Bruno. 2009. « La langue claire de Descartes », Rue Descartes 65, 20-34

Maingueneau, Dominique. 2015. La philosophie comme institution discursive, chap. 7 : « Langue et code langagier » (Limoges : Lambert-Lucas), 105-118

\subsection{Le texte philosophique}

Bordron, Jean-François. 1987. Descartes. Recherches sur les contraintes sémiotiques de la pensée discursive (Paris : PUF)

61 Jacques, Francis. 1987. «Le moment du texte ", Greisch, Jean (éd.). Le texte comme objet philosophique (Paris : Beauschesne), 15-85

62 Cossutta, Frédéric. 1995. "Pour une analyse du discours philosophique ", section III : "Anaphore textuelle, contraintes didactiques et systématicité dans l'Ethique de Spinoza ", Langages 119, 29-38 Sini, Carlo. 1995. Filosofia e scrittura (Roma-Bari : Sagittari Laterza) Bordron, Jean-François. 1998. « Discours philosophique et narrativité », Encyclopédie philosophique universelle, T. IV, ch. $\mathrm{n}^{\circ} 86,1532-1540$ Jacques, Francis. 1998. «L'ordre du texte ", Encyclopédie Philosophique Universelle, T. IV, ch. $\mathrm{n} 102,1761-1792$ Minazzi, Fabio (éd.). 2000. La scritura filosofica : Generi letterari, destinattari, finalità e forme della scrittura filosofica (Roma : Ministero della Pubblica Istruzione)

67 «La philosophie et ses textes ». 2001. Methodos 1, https://journals.openedition.org/ methodos $/ 30$

Clément, Bruno (éd.). 2005. « L'Écriture des philosophes », Rue Descartes 50

Jacques, Francis. 2007. L'arbre du texte et ses possibles (Paris : Vrin)

\subsection{Approches stylistiques et énonciatives}

Granger, Gilles Gaston. 1968. Essai d'une philosophie du style (Paris : Colin) 
Cahné, Pierre-Alain. 1980. Un autre Descartes. Le philosophe et son langage (Paris : Vrin)

Ali Bouacha, Magid. 1995. «De l'ego à la classe de locuteurs : lecture linguistique des Méditations ", Langages 119, 79-94

Ehlich, Konrad. 1995. «Manière de penser, manière d'écrire : la procédure phorique dans le texte hegélien ", Langages 119, 109-122

Philippe, Gilles. 1995. « Embrayage énonciatif et théorie de la conscience : à propos de L'être et le néant », Langages 119, 95-108

Lhomme, Alain. 1998. « Le style des philosophes ", Jean-François Mattéi (éd.). Le discours philosophique. Encyclopédie philosophique universelle (Paris : PUF), 1564-1583

Temmar, Malika. 2001. «Les stratégies discursives d'ostension de l'objet chez R. Descartes ", Gronemann, Claudia, Christiane Maaß, Sabine Peters \& S. Schrader (éds.) 2011. Körper und Schrift (Bonn : Romanistischer Verlag), 323-329

7 Temmar, Malika. 2003. «Effets de présence et ostension dans le discours phénoménologique merleau-pontyen: une approche discursive ", Studia Phaenomenologicsa, Romanian Journal for Phenomenlogy, 3/4, 40-67

8 Libris, Jean, Maryvonne Perrot et Jean-Jacques Wunenberger (éds.). 2004. Bachelard et l'écriture, Cahiers Gaston Bachelard (Dijon : Université de Bourgogne)

Curatolo, Bruno \& Jacques Poirier (éds). 2007. Le style des philosophes (Dijon : Éditions Universitaires de Dijon)

Adam. Jean-Michel. 2008. "La phrase dans le discours. Autour de quelques réénonciations du cogito cartésien ", O. Brand et al. (éds). Discours, diachronie, stylistique du français. Études en hommage à Bernard Combette (Bern : Peter Lang), 3-16

81 Temmar, Malika. 2009. «Les commentaires méta-énonciatifs dans le discours phénoménologique merleau-pontyen », Recherches linguistiques, 30, 70-82

Cohen-Halimi, Michèle. 2010. « Les tirets de Nietzsche », Ligne, $13: 1,35-47$

Delormas, Pascale. 2012. «Un cas particulier d'interdiscursivité : l'autocitation dans l'auto/bio/graphie de philosophe », Mur, Lorda \& Clara Ubaldina (éds.). Polifonía e intertextualidad en el diálogo (Madrid : Arco libros), 83-95

84 Angermuller, Johannes. 2013. Analyse du discours poststructuraliste. Les voix du sujet dans le langage chez Lacan, Althusser, Foucault, Derrida et Sollers (Limoges : Lambert Lucas)

Clément, Bruno. 2013. La Voix verticale - Essai sur la prosopopée (Paris : Belin)

Lhomme, Alain. 2017. «La fabrique du spéculatif. Essai d'analyse du $§ 36$ de Par delà le bien et mal ", José Aguilar, Lucie Cadet, Catherine Muller \& Véronique Rivière (éds.), L'enseignant et le chercheur au cœur des discours, des textes et des actions. Mélanges offerts à Francine Cicurel (Paris : Riveneuve éditions), 73-94

\subsection{Enonciation philosophique et fiction}

87 Lhomme, Alain. 2000. «Pensées fictives et fictions de l'imagination. Sur l'écriture de la fiction dans les Méditations métaphysiques de Descartes », Europe 849/850, 184-200 Clément, Bruno. 2005. Le récit de la méthode (Paris : Seuil) Clément, Bruno. 2008. "Descartes romancier, Proust philosophe », Actes de savoirs, 4 (Paris : PUF), 129-144 
Temmar, Malika. 2009 «La statue de Condillac dans le Traité des sensations », Alliage 65, 58-72 [En ligne : http://revel.unice.fr/alliage/index.html ?id =3373]

Temmar, Malika. 2013. Le recours à la fiction dans le discours philosophique (Limoges: Lambert-Lucas)

\subsection{La lecture du texte philosophique}

Cossutta, Frédéric. 1989. Eléments pour la lecture des textes philosophiques (Paris : Bordas)

Costa, Filipo (éd.). 1992. Il testo filosofico. Ermeneutica teoria e pratica (Palermo : l'epos)

Lhomme, Alain. 1998. «Le travail de la lecture », L'École des philosophes 4, «Les textes dans l'enseignement philosophique » (Lille : CRDP du Nord-Pas de Calais)

Cossutta, Frédéric. 1999. "La lettura dei testi filosofici : questioni di metodo », Fabio Minazzi (éd.). La scrittura filosofica: Generi letterari, forme e finalità della scrittura filosofica (Roma : Ministero della Pubblica Istruzione), 97-153

Cossutta, Frédéric. 2000. « Lire, élire les philosophes », entretien avec Francine Cicurel. Les textes et leur lecture, Cicurel, Francine (éd.). Études de linguistique appliquée (Paris: Didier Érudition), 373-384

Rey, Jean-Michel. 2005. «L'idée à la place du texte », Rue Descartes 50, 34-46

Ribard, Dinah. 2003. "Nom d'auteur et effets de lecture: L'entretien de Pascal avec Monsieur de Sacy, XVII ${ }^{\text {éme-XX }}{ }^{\text {ème }}$ siècle », XVII Siècle 219, 259-270

Cicurel, Francine. 2004. «Le lu et le lire ou l'espace de la lecture », Adam, Jean-Michel, Jean-Blaise Grize \& Magid Ali Bouacha (éds.) ». Texte et discours : catégories pour l'analyse (Dijon : Editions Universitaires de Dijon), 215-231

C Cicurel, Francine. 2015. "La mise en fiction du désespoir, l'éthos kierkegaardien ", Analyse du discours et dispositifs d'énonciation, Angermuller, Johannes \& Gilles Philippe (éds) Autour des travaux de Dominique Maingueneau (Limoges : Lambert-Lucas)

1 Ali Bouacha, Magid. 2017 (posthume). «Les écrits de Bachelard: entre fictivité et fiction ", Angermuller, Johannes \& Gilles Philippe (éds), Analyse du discours et dispositifs d'énonciation. Autour des travaux de Dominique Maingueneau (Limoges : Lambert-Lucas)

\section{Les genres de la philosophie}

\subsection{Généralités}

2 Cossutta, Frédéric. 1998 "Les genres en philosophie", Encyclopédie Philosophique Universelle, T. IV, chap. 85, 1512-1532

Maingueneau, Dominique. 2004. "Retour sur une catégorie: le genre », Adam, JeanMichel, Jean-Blaise Grize \& Magid Ali Bouacha (éds) Texte et discours : catégories pour l'analyse (Dijon : Editions Universitaires de Dijon), 107-118

4 Temmar, Malika. 2007. « Le genre philosophique aux carrefours des genres », Le Français aujourd'hui 159, « Le genre comme outil, objet, enjeu », 55-62

Maingueneau, Dominique. 2015. La philosophie comme institution discursive, chap. 4 «Scène d'énonciation et généricité » (Limoges : Lambert-Lucas), 65-80 


\subsection{Le dialogue philosophique et les genres dialogiques}


Laks, André. 2004 « "Qu'importe qui parle". Remarques sur l'anonymat platonicien », Cossutta (éd). 2004, 107-122

Lambert, Jean. 2004 « Le sens et les fonctions du dialogue dans l'œuvre scientifique de Galilée », Cossutta (éd.). 2004, 141-166

Lhomme, Alain. 2004. «L'éducation de Pamphile ou le théâtre de la raison sceptique : essai d'analyse des Dialogues sur la religion naturelle », Cossutta. (éd.). 2004, 207-236

Lhomme, Alain. 2004. «Le régime de l'hypothétique dans le Phédon de Platon », Adam, Jean-Michel, Jean-Blaise Grize \& Magid Ali Bouacha (éds), Texte et discours : catégories pour l'analyse (Dijon : Éditions universitaires de Dijon), 231-242

Ostrowiecki-Bah, Hélène. 2004. « Dialogue et libertinage », Cossutta (éd.). 2004, 167-182

Maingueneau, Dominique. 2006. «Le dialogue comme hypergenre », Guérin, Philippe (éd.). Le dialogue ou les enjeux d'un choix d'écriture (Rennes: P. U. de Rennes), 35-49

Spranzi, Marta. 2011. The Art of Dialectic between Dialogue and Rhetoric (Amsterdam Philadelphia : John Benjamins Publishing Company)

Cossutta, Frédéric. 2019. «Du dialogue à la "diatribe". Le cas des Diatribai d'Epictète/ Arrien ", Jean-Pierre \& Anne-Marie Favreau-Linder (éds.). La diatribe, un genre dialogique dans la philosophie antique? (Limoges, Lambert-Lucas), 65-86

\subsection{Formes brèves}

Cossutta, Frédéric. 2004. «Écriture poétique, écriture de la poétique : le sens de la formule chez G. Bachelard ", Bachelard et l'écriture, Cahiers Gaston Bachelard (Dijon : Centre Gaston Bachelard),191-205

Ronkhomovsky, Bernard. 2005. Lire les formes brèves (Paris : A. Colin)

Maingueneau, Dominique. 2012. Les phrases sans texte (Paris : A. Colin)

Cossutta, Frédéric \& Francine Cicurel (éds). 2014. Les formules philosophiques. Détachement, circulation, recontextualisation (Limoges : Lambert-Lucas)

Bordron, Jean-François. 2014. "Figures du détachement », Cossutta et Cicurel (éds.), 53-70

Cossutta, Frédéric. 2014. «Le rôle des formules dans le discours philosophique », Cossutta \& Cicurel (éds.), 71-108

Cicurel, Francine. 2014. "Mot d'ordre ou mot de passe: les formes textuelles de la transmissibilité en philosophie », Cossutta et Cicurel (éds.), 111-113

Lhomme, Alain. 2014. "Formules philosophiques et écriture formulaire», Cossutta \& Cicurel (éds.), 25-52

Rabatel, Alain, 2014. «Des formules aphoristiques (dans le Dictionnaire philosophique de Comte-Sponville) au service su sujet philosophant: coénonciation, surénonciation, sousénonciation », Cossutta \& Cicurel (éds.), 163-198

Temmar, Malika. 2014. «Formules, sentences, maximes: la glose en philosophie», Cossutta \& Cicurel (éds.), $131-140$

Maingueneau, Dominique. 2014. "La formule philosophique et ses commentaires", Cossutta \& Cicurel (éds), 199-220 


\section{Polémique et controverses}

144 Maingueneau, Dominique. 1984. Genèse du discours, chap. 4 "La polémique comme interincompréhension » (Liège : Mardaga)

Potte-Bonneville, Mathieu. 1992. «La violence dans le débat philosophique. La controverse Derrida/Searle », Vernant, Denis (éd.). Recherches sur la philosophie et le langage 14, «Du Dialogue » (Paris : Vrin), 219-248

146 Dascal, Marcello. 1995. "Observations sur la dynamique des controverses », Cahiers de linguistique française 17, 99-121

147 Dascal, Marcello. 1998. «La controverse en philosophie », Encyclopédie Philosophique Universelle T. IV, ch. $\mathrm{n}^{\circ} 90$ 1583-1604

148 Ali Bouacha, Magid. 2000. "L'AUTRE du sujet dans 'La recherche de la vérité par la lumière naturelle' de Descartes ", Ali Bouacha \& Cossutta (éds), 19-37

149 Bordron, Jean-François. 2000. « Res cogitans, Descartes et Brunelleschi », Ali Bouacha \& Cossutta (éds) (éds), 39-58

150 Cicurel, Francine. 2000. "Une mise en scène de la polémique: les dialogues philosophiques contemporains ", Ali Bouacha \& Cossutta (éds), 131-152

151 Cossutta, Frédéric \& Magid Ali Bouacha (éds.). 2000. La polémique en philosophie. La polémicité philosophique et ses mises en discours (Dijon : Éditions Universitaires)

152 Cossutta, Frédéric. 2000. "Typologie des phénomènes polémiques dans le discours philosophique », Ali Bouacha \& Cossutta (éds.) 167-207

153 Giolito, Christophe. 2000. «Le débat cartésien entre Alquié et Gueroult : controverse ou polémique ? ", Ali Bouacha \& Cossutta (éds.), 97-130

154 Lhomme, Alain. 2000. « Polemos et philia », Ali Bouacha \& Cossutta (éds.), 71-96

155 Maingueneau, Dominique. 2000. «Les deux ordres de contraintes de la polémique », dans Ali Bouacha \& Cossutta (éds.), 153-166

156 Cossutta, Frédéric. 2004. "Controversy and dialogic intersubjectivity ", Barotta, PierLuigi \& Marcelo Dascal (eds.). Controversies and Subjectivity (Amsterdam: John Benjamins), 127-156

157 Angenot, Marc. 2008. Dialogues de sourds. Trait de rhétorique antilogique (Paris : Mille et une nuits)

158 Maingueneau, Dominique. 2008. "Les trois dimensions du polémique », Gaudin-Bordes, Lucile \& Geneviève Salvan (éds.). Les registres. Enjeux stylistiques et visées pragmatiques (Louvain-la-Neuve : Academia-Bruylant), 107-118

159 Fotouhi, Khodayar. 2014. "Entre Foucault et Derrida : le sort d'un énoncé cartésien ", Cossutta, Frédéric \& Francine Cicurel (éds.). Les formules philosophiques. Détachement, circulation, recontextualisation (Limoges : Lambert-Lucas), 140-159 


\section{Les ressources et modes opératoires de la philosophie}

\subsection{Les concepts philosophiques}

\subsubsection{La mise en œuvre des concepts}

160 Cassirer, Ernst. 1977 [1910]. Substance et fonction, éléments pour une théorie du concept (Paris : Minuit).

161 Cossutta, Frédéric. 1989. Eléments pour la lecture des textes philosophiques (ch. 2 «Les concepts philosophiques", ch. 3 "La référence: du concept à l'exemple ») (Paris : Bordas)

162 Cotten, Jean-Pierre et Bernard Hufschmitt (éds). 2001. Repérer, formaliser, traduire les concepts philosophiques (Besançon : P. U. Franc-Comtoises)

163 Lhomme, Alain. 2005. «Entre concept et métaphore: existe-t-il une écriture spécifiquement philosophique? ", Rue Descartes 50, « L’Écriture des philosophes », 59-73 Blumenberg, Hans. 2017 [2007]. Théorie de l'inconceptualité (Paris : Editions de l'Eclat) Valette, Mathieu. 2010. "Des textes au concept. Propositions pour une approche textuelle de la conceptualisation", Després, Sylvie (éd.). Actes des $21^{e^{e} e s}$ Journées francophones d'Ingénierie des Connaissances (Publication de l'Ecole des Mines d'Alès), 5-16 (en ligne <hal-00491037>)

During, Elie. 2011. "Cartes et graphes : les usages du concept chez James et Bergson ", Madelrieux, Stéphane (éd.). Bergson et James : cent ans après (Paris : PUF)

Fasciolo, Marco (éd.). 2011. « Lexique et philosophie », Cahiers de lexicologie 99

Cools, Arthur, Walter Van Herck \& Koenraad Verrycken (éds). Metaphors in Modern and Contemporary Philosophy (Antwerpen : U. P. Antwerp), 53-82 Cossutta, Frédéric. 2013. "Philosophical Metaphors and Discourse Analysis», Cools, Arthur, Walter Van Herck \& Koenraad Verrycken (éds), Metaphors in Modern and Contemporary Philosophy (Antwerpen : University Press Antwerp), 53-82

Delormas Pascale. 2017. « Du concept au concetto. Manifestation discursive de la rupture ontologique dans De l'existence à l'existant de Levinas", Agular, José, Lucile Cadet, Catherine Muller \& Véronique Rivière (éds.). L'enseignant et le chercheur au cœeur des discours et des actions. Mélanges offerts à Francine Cicurel (Paris : Riveneuve Éditions), 55-72 Marras, Cristina. 2017. Les métaphores dans la philosophie de Leibniz (Limoges : LambertLucas)

Brière, Véronique. 2019. "L'invention des catégories chez Aristote: éléments pour penser la fabrication des concepts ", dans Cossutta (éd.) Cossutta, Frédéric. (éd.). (À paraître). La fabrique discursive des concepts philosophiques (Limoges : Lambert-Lucas) Delormas, Pascale. 2019. «Le concept, un intraduisible ? L'exemple de 'De l'existence à l'existant' de Levinas », Cossutta (éd.)

175 Lhomme, Alain. 2019. "Thématisation et reconfiguration textuelle des concepts », dans Cossutta (éd.).

Lhomme, Alain. 2019. « La reconfiguration textuelle des concepts », Cossutta (éd.) 
Lyons, John. 1969. Structural Semantics. An Analysis of Part of the Vocabulary of Plato (Oxford: Blackwell)

Maingueneau, Dominique. 2019. "Cartographie philosophique et extraction de concepts ", dans Cossutta (éd.)

Soulez Antonia. 2019. "La "descente ordinaire" des concepts selon Wittgenstein", Cossutta (éd.)

Vallespir, Mathilde. 2019. "Relire et réécrire le concept : le pharmakon comme anticoncept dans la Dissémination de Jacques Derrida », Cossutta (éd.)

\subsubsection{Lexiques, vocabulaires, dictionnaires, encyclopédies, index}

Auroux, Sylvain. 1990. « Avant propos ", Auroux, Sylvain (éd.). Les notions philosophiques, tome I, Encyclopédie philosophique universelle II (Paris : PUF), VII-XX

Cassin, Barbara. 2004. "Présentation », Cassin, Barbara (éd.). Vocabulaire européen des philosophies. Dictionnaire des intraduisibles (Paris : Le Robert-Seuil), XVII-XXIV

Savatovsky, Dan. (2007). «Le Vocabulaire philosophique de Lalande (1902-1903): lexicographie spécialisée ou prototerminogrie? », Langages 168, 39-52

Ducard, Dominique. 2019. «Terme, notion, concept. Le cas exemplaire du Vocabulaire de Lalande ", Cossutta, Frédéric (éd.). La fabrique discursive des concepts (Limoges : LambertLucas)

\subsubsection{Lexicométrie}

Robinet, André (éd.) 1973. "Etudes philosophiques et informatique», Revue internationale de philosophie 103

D’Iorio, Paolo (éd.). 2000. Hyper Nietzsche (Paris : PUF)

Favier, Laurence. 2004. "Analyse lexicométrique de La Psychanalyse du feu ", Jean Libris, Maryvonne Perrot et Jean-Jacques Wunenberger (éds), Bachelard et l'écriture, Cahiers Gaston Bachelard (Dijon : Université de Bourgogne), 301-3018

Valette, Mathieu 2010. "Propositions pour une lexicologie textuelle», Blumenthal, Peter \& Salah Mejri (éds.). Zeitschrift für Französische Sprache und Literatur 37, "Les configurations du sens ", 171-188

Rastier, François. 2011. La Mesure et le Grain. Sémantique de corpus (Paris : Champion)

\subsection{La validation des énoncés philosophiques : argumentation, persuasion, démonstration}

Perelman, Chaïm \& Lucie Olbrechts-Tyteca. 1952, Rhétorique et philosophie. Pour une théorie de l'argumentation philosophique (Paris : PUF)

Perelman, Chaïm \& Lucie Olbrechts-Tyteca 2008 [1958]. Traité de l'argumentation. La nouvelle rhétorique (Bruxelles : Editions de l'Université de Bruxelles)

Toulmin, Stephen. 1958. The uses of Arguments (Cambridge : Cambridge U. P.)

Passmore, John. 1961. Philosophical Reasoning (London : Duckworth)

Grize, Jean-Blaise (éd.). 1984. Sémiologie du raisonnement (Berne : Peter Lang) 
Cossutta, Frédéric.1989. Eléments pour la lecture des textes philosophiques, chap. 5 «Stratégie discursive et argumentation » (Paris : Bordas), 139-206

Ali Bouacha, Magid. 1993. «Enonciation Argumentation et discours. Le cas de la généralisation », Semen 8, 43-61

Bouvier Alban. 1995. L'argumentation philosophique. Etude de sociologie cognitive (Paris: PUF)

Cossutta, Frédéric (éd.). 1996. Descartes et l'argumentation philosophique (Paris : PUF)

Boyer, Alain \& Georges Vignaux (éds. ). 1998. « Argumentation et rhétorique », Hermès 15

Cossutta, Frédéric. 2004. « Neutralisation du point de vue et stratégies argumentatives dans le discours philosophique ", Ruth Amossy \& Roselyne Koren (éds.), Argumentation et prises de position : pratiques discursives, Semen 17, 81-97

Thibaudeau, Victor. 2006. Principes de logique. Définition, Énonciation, Raisonnement (Québec : PUL)

Plantin, Christian. 2016. Dictionnaire de l'argumentation. Une introduction aux études d'argumentation (Lyon : ENS Editions)

\section{7. Études collectives d'un auteur ou d'un même texte dans une perspective discursive}

\subsection{Bergson}

Ali Bouacha, Magid. 1998. «La réfutation dans "Le possible et le réel” ", Cossutta (éd.), 201-217

Bordron, Jean-François. 1998. «Bergson et les images. L'iconicité de la pensée dans Le possible et le réel », Cossutta (éd.), 159-182

Cossutta, Frédéric (éd.). 1998. Lire Bergson : « le possible et le réel » (Paris : PUF)

Cossutta, Frédéric. "L'œuvre de Bergson, une “création continue d'imprévisible nouveauté"? ", Cossutta (éd.), 49-100

Giolito, Christophe. 1998. «L'impossible et le réel. Sur la représentation », Cossutta (éd.), 139-158

Lhomme, Alain. 1998. " Formuler l'informulable, analyse d'un paradoxe pragmatique », Cossutta (éd.), 101-138

Maingueneau, Dominique. 1998. " "Le possible et le réel” de Bergson : quel genre de texte? ", Cossutta (éd.), 29-48

10 Philippe, Gilles. 1998. "Théorie du lexique et pratiques d'écriture: les gloses métadiscursives dans "Le possible et le réel” ", Cossutta (éd.), 183-200

\subsection{Derrida}

1 Angermuller, Johannes. 2015. «Le déconstructivisme derridien. De la subjectivité dans les discours philosophique ", Maingueneau \& Vallespir (éds), 93-102 


\section{AUTEURS}

\section{FRÉDÉRIC COSSUTTA}

CEDITEC, Paris-Est Val de Marne

DOMINIQUE MAINGUENEAU

Sorbonne Université

Argumentation et Analyse du Discours, 22 | 2019 